\title{
Avaliação de Séries de Velocidade do Vento de Produtos de Reanálises Climáticas para o Brasil
}

\author{
Cesar Augusto Crovador Siefert ${ }^{1}$ (D), Nicolas Dombrowski Netto ${ }^{1}$, \\ Fernando Helmuth Syring Marangon ${ }^{1}$, Gilson Bauer Schultz ${ }^{1}$, Luan Marcus dos Reis Silva ${ }^{1}$, \\ Thiago Henriques Fontenelle ${ }^{2}$, Irani dos Santos ${ }^{1}$ \\ ${ }^{I}$ Departamento de Geografia, Universidade Federal do Paraná, Curitiba, PR, Brasil. \\ ${ }^{2}$ Agência Nacional de Águas e Saneamento Básico, Brasília, DF, Brasil.
}

Recebido em: 4 de Março de 2021 - Aceito em: 7 de Maio de 2021

\begin{abstract}
Resumo
A principal limitação para estudos climáticos em escala regional no Brasil é a disponibilidade de dados meteorológicos observados em termos de quantidade, qualidade e da sua distribuição espacial. Dados de velocidade de vento de reanálises climáticas são utilizadas para diversos estudos de modelagem climatológica e hidrológica, porém a qualidade destes sobre o território brasileiro ainda é desconhecida considerando produtos de reanálises em escala diária e resoluções espaciais $<1^{\circ}$. Neste trabalho, a performance de 3 produtos de reanálises (ERA5, GLDAS 2.1 e MERRA-2) foi avaliada para a representação de dados de velocidade do vento superficiais em escala diária a partir de dados observacionais de 521 estações meteorológicas para o período de 2000-2018. A acurácia das reanálises foi avaliada considerando a sua capacidade em reproduzir a tendência, variabilidade média e sazonal e heterogeneidade espacial das séries observadas. Os conjuntos de reanálises analisados apresentam limitações e incertezas para simulação dos dados considerando a tendência e dispersão diária das séries observadas. Observa-se uma tendência de superestimativa das velocidades diárias das reanálises para as zonas climáticas brasileiras. A análise comparativa indica que os dados do ERA5 apresentaram uma maior acurácia para as zonas climáticas do país em termos de tendências médias e sazonalidades.
\end{abstract}

Palavras-chave: velocidade do vento, variáveis meteorológicas, reanálises climáticas, acurácia.

\section{Evaluation of the Wind Speed Time Series from Climate Reanalysis for Brazil}

\begin{abstract}
A major limitation of regional climate studies in Brazil is related to the availability of meteorological datasets in terms of data quality, quantity, and its spatial density distribution. Wind speed data from climate reanalysis has been used as input in several studies for climate and hydrological modeling however the quality of these datasets over Brazilian territory remains unknown for daily datasets at spatial resolutions $<1^{\circ}$. In this study, the performances of 3 sets of reanalysis data (ERA5, GLDAS 2.1, and MERRA-2) are assessed in reproducing surface wind speed on a daily scale for the period of 2000-2018 using data from 521 meteorological stations as a baseline. The reanalysis's accuracy is evaluated by comparing simulated trends, mean and seasonal variability, and spatial heterogeneity of observation data. All 3 sets show several uncertainties in simulated wind speed concerning its trends and dispersion in daily intensity compared to observed series. Our results show the wind speed derived from reanalysis presents a significant increase in trends on daily wind speed throughout the Brazilian climate zones. From the results of the comparative analysis, the ERA5 dataset simulated more accurately wind speed daily trends and seasonality over the climate zones in Brazil.
\end{abstract}

Keywords: wind speed, meteorological variables, climate reanalysis, accuracy.

Autor de correspondência: Cesar Augusto Crovador Siefert, cesarsiefert@ufpr.br. 


\section{Introdução}

A rede meteorológica nacional operada pelo Instituto Nacional de Meteorologia (INMET) apresenta variação significativa em termos de densidade de estações por unidades federativas. Por exemplo, unidades federativas como Minas Gerais, Bahia, Rio Grande do Sul e São Paulo concentram cerca de $1 / 3$ das 789 estações meteorológicas em operação em $20 \%$ do território nacional. Distrito Federal, Rio de Janeiro, Sergipe e Alagoas, comparativamente, possuem as maiores densidades de estações meteorológicas em seus territórios. Por outro lado, 9 unidades (i.e. Roraima, Rondônia, Amazonas, Amapá, Pará, Acre, Mato Grosso, Maranhão e Tocantins), correspondem a $60 \%$ do território nacional e possuem uma densidade de estações meteorológica inferior a $1 / 10.000 \mathrm{~km}^{2}$.

Este cenário de escassez de dados em algumas regiões conduz a dificuldades e incertezas no desenvolvimento de malhas interpoladas a partir de dados observados (Amini et al., 2018), independente da complexidade ou da quantidade de informação adicional utilizada no processo de interpolação (Newman et al., 2015). Assim, reanálises climáticas são produtos que têm gerado interesse cada vez maior (Hersbach et al., 2020) em função de sua aplicabilidade e consistência ao combinar uma ampla variedade de dados observados em superfície ou detectados remotamente dentro de um modelo numérico dinâmico de base física (Tarek et al., 2020) em escala continental ou global.

Estes produtos são constituídos de matrizes de dados, em resolução temporal e espacial diversa, gerados pela combinação entre dados observados e esquemas de assimilação do modelo numérico de previsão de tempo utilizado e posterior interpolação (Dee et al., 2016; Limberger e Silva, 2018). Ao combinar observações, dados de sensoriamento remoto e modelos, as reanálises fornecem séries consistentes das essential climate variables (ECVs) e buscam garantir a consistência e coerência na representação dos principais ciclos do sistema terrestre (p. ex. água e energia) (Hersbach et al., 2020). Produtos de reanálises se tornam ferramentas essenciais para a compreensão da evolução atmosférica (Ramon et al., 2019) e tem sido utilizado como base para modelagens diversas para descrição da dinâmica e tendências das condições atmosféricas (Bengtsson et al., 2004; Fang et al., 2018; Mukherjee et al., 2018) ou hidrológicas (Chen et al., 2018; Uniyal et al., 2019; Tarek et al., 2020), sobretudo devido a sua continuidade temporal e espacial (Yu et al., 2019).

Considerando que diversos centros meteorológicos possuem os seus conjuntos de reanálises, via de regra, a partir de seu próprio modelo, os produtos apresentam diferenças entre si, levando a realização de estudos de comparação para diversas variáveis para avaliação de sua performance com relação a dados observados ou da sua adequabilidade para o uso (p.ex. Lindsay et al., 2014; Kaiser-Weiss et al., 2015; Ramon et al., 2019, Yu et al., 2019;
Delhasse et al., 2020) em função dos possíveis impactos de dados climáticos como input em modelagens diversas. Devido a ampla disponibilidade de produtos em escalas espaciais e temporais distintas e suas aplicações específicas, a avaliação da adequabilidade ao uso de um determinado produto deve compreender as limitações/potencialidades de uso, ajuste dos dados a dados observados e tolerância a incerteza nas aplicações (Kaiser-Weiss et al., 2015).

Embora em menor escala que dados de temperatura e precipitação (Pryor et al., 2009), dados brutos de velocidade do vento (e seus componentes, $u$ e $v$ ) derivados dos produtos de reanálises têm sido amplamente utilizados e avaliados em aplicações variadas e sob distintas condições geográficas devido à falta de séries observacionais longas e homogêneas (Cannon et al., 2015; Ramon et al., 2019). $\mathrm{Na}$ Alemanha, Kaisser-Weiss et al. (2015) comparou dados de velocidade do vento próximo da superfície em 210 estações com 3 conjuntos (COSMO-REA6, ERA-Interim e ERA-20C), indicando distribuições de frequência semelhantes dado o ajuste entre os parâmetros de Weibull derivados das séries de médias diárias. Na China, Yu et al. (2019) avaliou 5 conjuntos de reanálises (NCEP-2, MERRA, JRA-55, ERA-Interim e 20CR) em base mensal, indicando uma performance razoável na reprodução da distribuição espacial e uma subestimativa na intensidade em diversas regiões do país, além da limitação em reproduzir a variabilidade anual. Zhang et al. (2019) utilizou dados do JRA-55 e do ERA-Interim para avaliação das tendências da velocidade de vento próxima da superfície na China, indicando que o JRA-55 obteve a melhor performance ao reproduzir a dinâmica e variabilidade espacial dos componentes do vento. Paredes et al. (2018) utilizou dados brutos do ERA-Interim (velocidade do vento, temperatura e radiação líquida) para estimativa da evapotranspiração de referência $\left(\mathrm{ET}_{0}\right)$ pelo método de PenmanMonteith propondo um método de correção de desvios no resultado a partir dos valores obtidos de $\mathrm{ET}_{0}$ para dados observados.

Para o Brasil, Micalichen e Dias (2018) compararam dados de 17 estações meteorológicas no estado de Minas Gerais com o CFSV2 do NCEP/NCAR, não indicando ajuste entre a variabilidade observada e a reproduzida. Gilliland e Keim (2017) avaliaram as tendências dos dados de velocidade e componentes do vento no país entre 1980-2014 utilizando 91 estações e 3 produtos de reanálise com resolução espacial $>0,75^{\circ}$ (NCEP/NCAR, NCEP-DOE e ERA-Interim), relatando diferenças entre velocidades médias e tendências entre as estações in situ e os produtos avaliados.

Este trabalho possui como objetivo realizar a avaliação da qualidade dos dados de velocidade do vento próximo a superfície para 3 produtos de reanálises amplamente utilizados: i) ERA5 (CCCS, 2017), fornecido pela European Centre for Medium-Range Weather 
Forecasts (ECMWF) Copernicus Climate Change Service (CCCS), ii) Global Land Data Assimilation System 2.1 (GLDAS 2.1) (Rodell et al., 2004; Beaudoing e Rodell, 2020), fornecido pela Goddard Earth Sciences Data and Information Services Center (GES DISC) da National Aeronautics and Space Administration (NASA), iii) Modern-ERA Retrospective Analysis for Research and Applications (MERRA-2) (Gelaro et al., 2017), fornecido pelo Global Modeling and Assimilation Office (GMAO) da NASA, em base diária e compreendendo o período de 2000-2018.

Esta escolha foi limitada a disponibilidade de produtos de reanálises de velocidade de vento e/ou seus componentes com resolução temporal diária, resolução espacial $<1^{\circ}$ e série $>10$ anos. A qualidade destes dados de reanálises sobre o território brasileiro ainda permanece desconhecida, sobretudo ao considerar as escalas diárias e em resoluções espaciais inferiores a $1^{\circ} \mathrm{e}$ a disponibilidade de séries de dados observados para o país. A avaliação foi realizada considerando dados observacionais da rede de dados meteorológicas do Brasil como base de comparação aos produtos de reanálise nos termos de características estatísticas gerais entre as séries, distribuição de frequência e sua variabilidade temporal.

\section{Material e Métodos}

\subsection{Dados observados}

Os dados de velocidade do vento utilizados neste estudo foram adquiridos e disponibilizados pelo INMET.

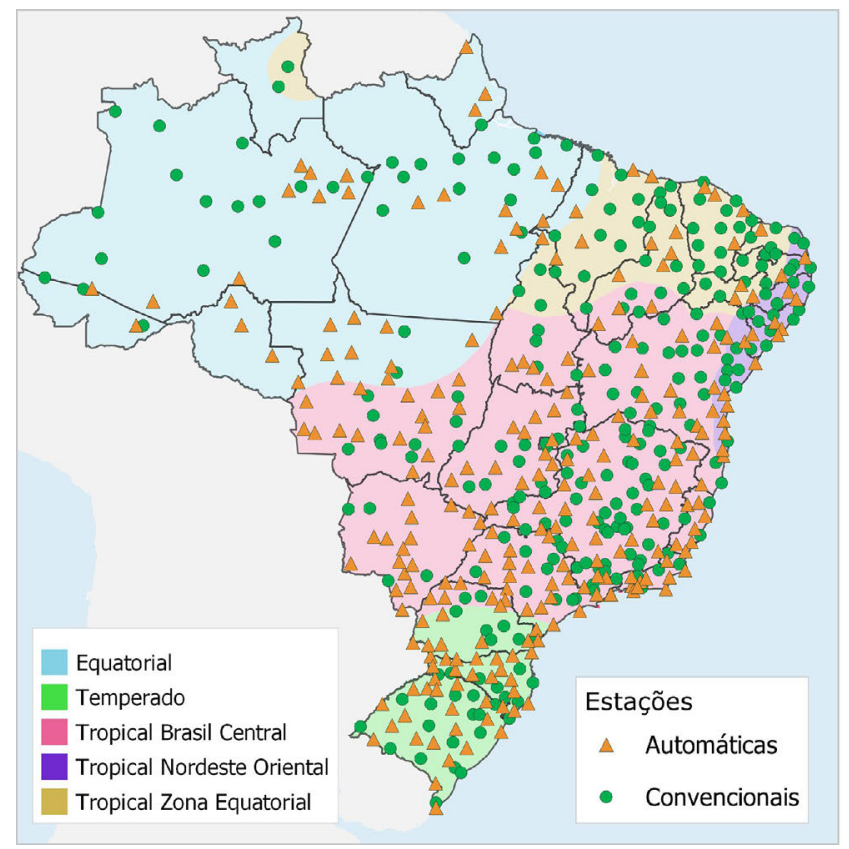

Foram utilizadas as séries brutas disponíveis entre o período de 1/1/2000 até 31/12/2018 de um total de 521 estações com o equivalente a 48 meses de dados em escala diária, sendo 260 convencionais e 261 automáticas. De acordo com as zonas climáticas brasileiras de Nimer (1979), 264 estações localizam-se na zona Tropical Brasil Central (TBC), 73 na zona Equatorial (E), 77 na zona Temperada (T), 65 na zona Tropical Zona Equatorial (TZE) e 42 na zona Tropical Nordeste Oriental (TNO). A distribuição espacial das estações meteorológicas utilizadas neste estudo e o número de estações com dados no respectivo ano são apresentados na Fig. 1 .

As estações convencionais do INMET adquirem dados de variáveis meteorológicas (p.ex. velocidade média do vento, umidade relativa do ar, insolação, temperatura, entre outros) 3 vezes ao dia (00:00, 12:00 e 18:00 UTC) enquanto as estações automáticas disponibilizam dados integralizados em resolução horária. Para a análise realizada, os dados em escala horária foram convertidos para médias diárias simples. De acordo com Santos e Silva (2013), os dados de velocidade do vento são coletados seguindo o padrão de altura estabelecido $(10 \mathrm{~m})$ pela Organização Meteorológica Internacional (World Meteorological Organization - WMO). Considerando a aplicabilidade dos dados de vento em modelos para estimativa de evapotranspiração de culturas, os dados observados a $10 \mathrm{~m}$ de altura foram convertidos para $2 \mathrm{~m}$ conforme proposto em Allen et al. (1998) a partir de um ajuste logarítmico do gradiente de vento em altura, utilizando como fator de correção um coeficiente de rugosidade para uma superfície de grama curta.

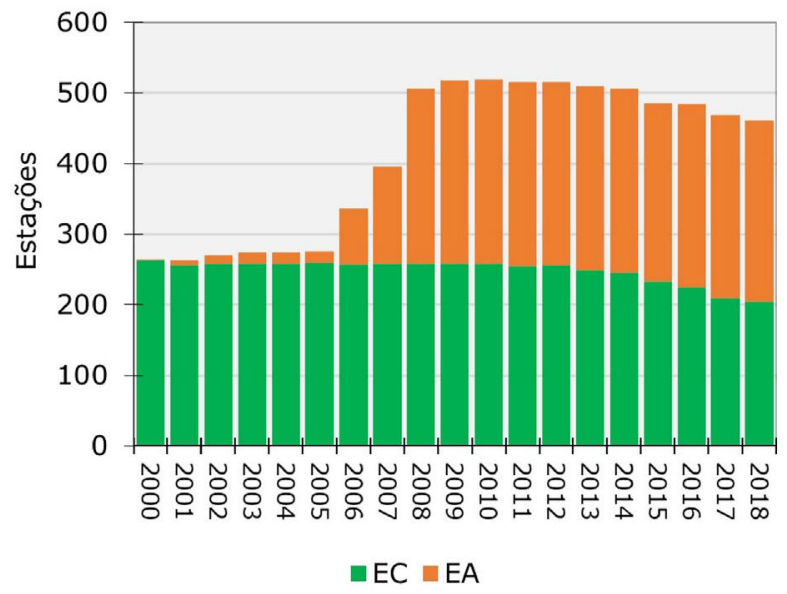

Figura 1 - Localização das estações meteorológicas utilizadas em relação as zonas climáticas e número de estações por ano (EA - estações automáticas, EC - estações convencionais). 


\subsection{Reanálises climáticas}

Três conjuntos de dados que representam o estadoda-arte das reanálises climáticas foram considerados neste estudo: ERA5, GLDAS 2.1 e MERRA-2. Destaca-se que nenhum dos produtos de reanálises avaliados assimila dados superficiais de velocidade de vento a partir de medições em superfície terrestre, o que pode causar falhas de representação devido a variabilidade meteorológica local (Ramon et al., 2019).

Os dados do ERA5, a $5^{\text {a }}$ geração de produtos de reanálises disponibilizado pelo ECMWF e substituto do ERA-Interim, são baseados no Integrated Forecasting System (IFS) Cy41r2, um sistema numérico de previsão meteorológica de alta resolução (CCCS, 2017). Os outputs em resolução horária, resolução horizontal de $\sim 30 \mathrm{~km}$ e 137 níveis verticais abrangendo a superfície da Terra até $0,01 \mathrm{hPa}$ capturam detalhes mais sutis dos fenômenos atmosféricos do que nas reanálises globais anteriores de baixa resolução (HERBSACH et al., 2020).

O GLDAS 2.1 é um dos componentes do projeto NASA Global Land Data Assimilation System Version 2 (GLDAS-2) que prevê um modelo de assimilação combinado baseado em dados observacionais (satélites e terrestres), modelos meteorológicos como variáveis de ajuste (p. ex. NOAA Climate Prediction Center Merged Analysis of Precipitation (CMAP), entre outros) e em técnicas de modelagem da superfície para geração de estados atmosféricos e fluxos de energia e água a partir da superfície (RODELL et al., 2004).

O Modern-Era Retrospective Analysis for Research and Applications, versão 2 (MERRA-2), produzido pela NASA Global Modeling and Assimilation Office (GMAO) é o sucessor do MERRA, assimilando atualizações do sistema Goddard Earth Observation System (GEOS) para as variáveis de circulação atmosférica (AGCM), oceânica (OGMC), modelo acoplado atmosfera-oceano (AOGCM) e ciclos químicos (CTM). O MERRA-2 disponibiliza um produto para análise climática contínua de modo intermediário, englobando desenvolvimentos recentes em termos de modelagem de assimilação de dados e parte do desenvolvimento do sistema integrado de análise terrestre (Gelaro et al., 2017).
A Tabela 1 lista as principais características com relação ao período disponível, resolução espacial e temporal e variáveis utilizadas para análise.

A série de dados estimada por cada um dos produtos avaliados foi obtida pela extração dos valores da célula em que cada estação está localizada. Para a obtenção das séries brutas de velocidade do vento para os produtos ERA5 e GLDAS 2.1 foi compilado um código em Python no Google Earth Engine (Gorelick et al., 2017) para extração das respectivas variáveis para o período 2000-2018 na resolução temporal diária (ERA5) e $3 \mathrm{~h}$ (GLDAS 2.1) para os pixels em que cada estação está localizada e posterior conversão para médias diárias (GLDAS 2.1). Para os dados do MERRA-2, o acesso aos dados ocorreu pelo repositório da GES DISC, realizando a extração das variáveis para os pontos de interesse das estações meteorológicas em software de geoprocessamento.

Para os dados do ERA5 e MERRA-2 foi necessária o cálculo da velocidade do vento ( $w s, \mathrm{em} \mathrm{m} / \mathrm{s})$ a partir dos componentes $u$ (componente paralelo ao eixo- $x$ - longitudinal) e $v$ (componente paralelo ao eixo-y - latitudinal) utilizado a equação $w s=\sqrt{u^{2}+v^{2}}$. Os dados de velocidade de vento dos produtos de reanálises a $10 \mathrm{~m}$ (i.e. ERA5 e GLDAS 2.1), a exemplo dos dados das estações meteorológicas, foram convertidos para $2 \mathrm{~m}$ conforme equação proposta em Allen et al. (1998):

$$
\begin{aligned}
u_{2} & =u_{z} \frac{4.87}{\ln (67.8 z)-5.42} ; \\
u_{2} & =u_{10} \frac{4.87}{\ln (67.8 \times 10)-5.42} ; \\
& =0.74795 u_{10} .
\end{aligned}
$$

\section{3. Índices para avaliação dos produtos de reanálises}

A performance dos dados de velocidade de vento obtido nos 3 produtos de reanálise foi avaliada com o objetivo de indicar se os dados são consistentes com relação aos dados observados a partir dos seguintes critérios de qualidade do ajuste: erro médio absoluto $(M A E)$, raiz do erro quadrático médio normalizada (NRMSE), coeficiente de correlação de Pearson ( $r$ e Bias, conforme equações apresentadas abaixo.

Tabela 1 - Resumo das características dos 3 produtos avaliados.

\begin{tabular}{lccc}
\hline & ERA5 & GLDAS 2.1 & MERRA-2 \\
\hline Fornecedor & ECMWF & NASA GES DISC & NASA GMAO \\
Período disponível & 1979 -presente & 2000-presente & 1980 -presente \\
Variáveis & Componentes $u$ e $v(10 \mathrm{~m})$ & Wind_f_inst $(10 \mathrm{~m})$ & Componentes $u$ e $v(2 \mathrm{~m})$ \\
Resolução temporal & $1 \mathrm{~h}$ & $3 \mathrm{~h}$ & $3 \mathrm{~h}$ \\
Resolução espacial & $0,25^{\circ} \times 0,25^{\circ}$ & $0,25^{\circ} \times 0,25^{\circ}$ & $0,5^{\circ} \times 0.625^{\circ}$ \\
Referência & $\mathrm{CCCS}, 2017$ & Beaudoing e Rodell, 2020 & Gelaro et al., 2017 \\
\hline
\end{tabular}




$$
\begin{gathered}
\text { MAE }=\frac{1}{n} \sum_{i=1}^{n}\left|x_{r}-x_{o}\right| \\
\text { NRMSE }=\frac{\left(\frac{\sum_{i=1}^{n}\left(x_{o}-x_{r}\right)^{2}}{n}\right)^{0,5}}{x_{\text {omáx }}-x_{\text {omin }}} \\
r=\sum\left(x_{o}-\bar{x}_{o}\right) \frac{\left(x_{r}-\overline{x_{r}}\right)}{\sqrt{\sum\left(x_{o}-\bar{x}_{o}\right) 2 \sum\left(x_{r}-\overline{x_{r}}\right) 2}} \\
\text { Bias }=\left[\frac{\sum_{i=1}^{n} x_{r}}{\sum_{i=1}^{n} x_{o}}-1\right]
\end{gathered}
$$

onde $x_{o}$ é a velocidade do vento $(\mathrm{m} / \mathrm{s})$ medida na estação, $x_{r}$ é a velocidade do vento estimada pelo produto de reanálise, $n$ é o número de observações.

$\mathrm{O} M A E$ indica a diferença média absoluta entre os dois conjuntos de dados, sendo que o valor é dado na mesma unidade dos conjuntos avaliados (i.e. $\mathrm{m} / \mathrm{s}$ ). O NMRSE, isto é, o RMSE normalizado pela diferença entre os valores máximos e mínimos do conjunto de dados observados, é mais apropriado para representação da performance de modelos do que o $M A E$ pois atribui um maior peso para desvios maiores. $\mathrm{O}$ valor é sempre positivo e um valor igual a zero indica o ajuste perfeito. O coeficiente de correlação de Pearson ( $r$ ) indica o grau de correlação entre os dois conjuntos de dados avaliando a capacidade do modelo de simular a variância dos dados observados sendo representado por valores entre -1 e 1 , onde 1 indica $o$ ajuste perfeito, sendo que, em geral, para uma relação linear positiva adota-se $r<0,5$ como ajuste fraco, moderado entre 0,5 e 0,7 e forte para valores de $r>0,7$. O Bias avalia a tendência que a média dos valores dos produtos de reanálise possuem em relação aos dados observados, sendo zero o valor ideal como indicativo de ajuste ótimo.
Como forma de avaliação da distribuição de frequência das séries, foi avaliado o ajuste obtido entre os parâmetros de Weibull das séries das reanálises e das séries observadas. Séries históricas de velocidade de vento tendem a se ajustar a uma função de distribuição de probabilidade do tipo Weibull, com dois parâmetros ( $k$ - parâmetro adimensional da forma do histograma e $c$ parâmetro da escala da distribuição, com a mesma unidade de ws), com aceitável nível de acurácia ao representar a variabilidade inerente dos regimes de vento (Carta et al., 2009; Angelis-Dimakis et al., 2010), inclusive para séries de dados no país (Rocha et al., 2012; Pes et al., 2017). Para a estimativa dos parâmetros $k$ e $c$ das séries observadas e das reanálises foi adotado o método empírico conforme utilizado em Rocha et al. (2012) e Sedghi et al. (2015), expresso pelas seguintes equações:

$$
\begin{aligned}
& k=\left(\frac{\sigma}{\bar{v}}\right)^{-1,086} \\
& c=\frac{\bar{v}}{\Gamma\left(1+\frac{1}{k}\right)}
\end{aligned}
$$

onde $\bar{v}$ é a velocidade média do vento $(\mathrm{m} / \mathrm{s})$ e $\sigma$ é o desvio padrão da série.

\section{Resultados e Discussão}

\subsection{Diferenças em termos de velocidades médias diárias mínimas, médias e máximas}

As velocidades médias de velocidade de vento a $2 \mathrm{~m}$ para as 521 estações meteorológicas analisadas com relação aos dados das reanálises são apresentadas na Fig. 2. Em termos gerais, as velocidades médias apresentam uma tendência de superestimativa com relação as séries observadas, evidenciando um ajuste moderado (ERA5 e GLDAS 2.1) a fraco (MERRA-2) com as séries das reanálises.
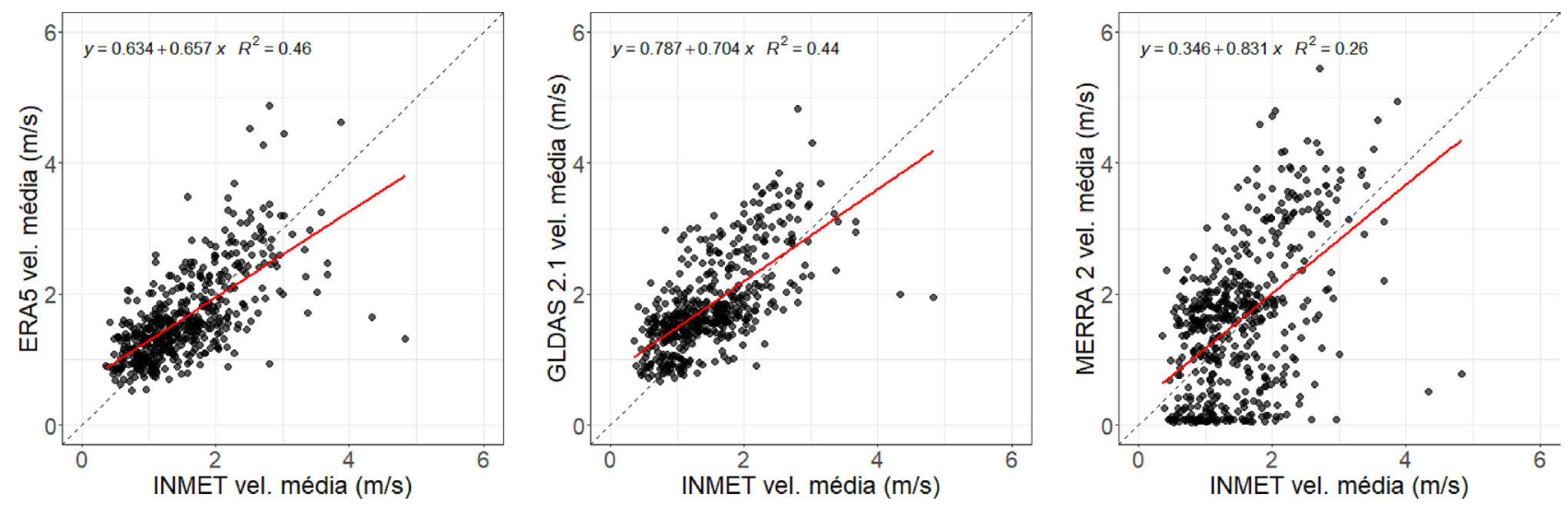

Figura 2 - Velocidade média do vento a $2 \mathrm{~m}(\mathrm{~m} / \mathrm{s})$ observada e dos produtos de reanálises. 
Uma possível limitação da comparação direta entre os produtos das reanálises e os dados observados está no fato de que os dados medidos são instantâneos. Por outro lado, os valores das reanálises representam a média da variável em um intervalo de tempo. Esta condição impacta diretamente a comparação dos valores mínimos e máximos observados e estimados pelos produtos de reanálises. Embora haja maior frequência de valores zero nas séries medidas, nas reanálises somente haverá valores iguais a zero quando esta condição se repetir em todo o intervalo de tempo considerado. Da mesma maneira, a ocorrência de valores máximos (p. ex.: acima de $5 \mathrm{~m} / \mathrm{s}$ ) nos dados observados está associada a medições instantâneas em rajadas de vento. Como os dados oriundos das reanálises representam a média do intervalo de tempo, a tendência é que, nestes casos, os valores sejam inferiores aos obtidos in situ.

Esta limitação na representação de velocidades de vento máximas também é relatada em Rose e Apt (2016) que indicam que os maiores erros nas reanálises ocorrem em estações com as maiores velocidades médias de vento. Destaca-se que a frequência e distribuição das velocidades de vento superficiais nos produtos de reanálise é impactada pelo uso do solo e condições de contorno utilizados na simulação que faz com que as condições locais se afastem das condições gerais atmosféricas. Isso pode indicar limitações na reprodução de fatores locais para a correta representação dos dados observados, como por exemplo, em função do modelo topográfico adotado, uso do solo e rugosidade do terreno no entorno das estações meteorológicas, conforme observado Gilliand e Keim (2017).

As velocidades máximas médias diárias de vento nos dados observados variam entre $1,5 \mathrm{~m} / \mathrm{s}-29,9 \mathrm{~m} / \mathrm{s}$ para $\mathrm{o}$ período 2000-2018. Para as reanálises, as velocidades máximas simuladas apresentam, em geral, uma tendência de subestimativa sobretudo com relação aos valores máximos para o país (i.e. estações convencionais de Rio Grande/RS, Areia/PB, Quixeramobim/CE, São Joaquim/SC, Santa Vitória do Palmar/RS e estação automática em São José dos Ausentes/RS, com velocidades máximas observadas acima de $15 \mathrm{~m} / \mathrm{s}$ ), variando entre $1,9 \mathrm{~m} / \mathrm{s}-11,3 \mathrm{~m} / \mathrm{s}$ (ERA5), $1,4 \mathrm{~m} / \mathrm{s}-11,8 \mathrm{~m} / \mathrm{s}$ (GLDAS 2.1) e $0,2 \mathrm{~m} / \mathrm{s}-$ $14,2 \mathrm{~m} / \mathrm{s}$ (MERRA-2).

As velocidades médias mensais para cada conjunto de dados foram classificadas em 4 grupos (0-2, 2-4, 4-5 e $>5 \mathrm{~m} / \mathrm{s}$ ) para avaliação geral da distribuição de frequência (Tabela 2). A diferença entre a representatividade dos valores de velocidade do vento obtidos nas estações e estimados nas reanálises pode ser verificada na Tabela 2 .

Em termos absolutos, os dados observados e do ERA5 indicam que cerca de $80 \%$ das médias mensais encontram-se entre $0-2 \mathrm{~m} / \mathrm{s}$, sendo que o ERA5 apresenta ainda uma ligeira superestimativa na faixa de $2-4 \mathrm{~m} / \mathrm{s}$. Estes resultados indicam que os ventos que predominam superficialmente no Brasil podem ser considerados como
Tabela 2 - Frequência das velocidades médias mensais a $2 \mathrm{~m}(\mathrm{~m} / \mathrm{s})$.

\begin{tabular}{lcccc}
\hline & $0-2$ & $2-4$ & $4-5$ & $>5$ \\
\hline INMET & $78,2 \%$ & $21,0 \%$ & $0,7 \%$ & $0,1 \%$ \\
ERA5 & $76,7 \%$ & $22,3 \%$ & $0,7 \%$ & $0,3 \%$ \\
GLDAS 2.1 & $70,4 \%$ & $28,3 \%$ & $1,2 \%$ & $0,1 \%$ \\
MERRA-2 & $69,4 \%$ & $26,2 \%$ & $3,7 \%$ & $0,7 \%$ \\
\hline
\end{tabular}

lentos e suaves. As maiores frequências de valores acima de $4 \mathrm{~m} / \mathrm{s}$ encontram-se nas estações nas zonas Temperado e Tropical Brasil Central, concentradas no período primavera - verão, sendo superestimadas no e GLDAS 2.1 e MERRA-2 e apresentando concordância com os dados do ERA5. Os maiores valores (> $5 \mathrm{~m} / \mathrm{s})$, embora pouco representativos no contexto geral das séries observadas ( $<1 \%$ dos meses), não são representados corretamente no ERA5 e no MERRA-2 em função de uma superestimativa nas médias mensais.

Dentre as 4 estações do ano, em concordância com os dados observados os valores médios de velocidade do vento a $2 \mathrm{~m}$ para os produtos de reanálise são sistematicamente maiores nos meses de primavera. Para as estações na zona Tropical Nordeste Oriental, as maiores velocidades médias de vento também ocorrem nos meses de verão. Este padrão não foi representado pelos produtos analisados, embora observe-se valores de velocidade média próximos com desvio inferior a $5 \%$ nas duas estações do ano para o GLDAS 2.1 e MERRA-2.

Os menores valores são observados nos meses de outono com exceção das estações na zona TNO que apresenta menores valores no inverno, sendo parcialmente reproduzidos pelos 3 produtos analisados. Gilliland e Keim (2017) indicam que a variabilidade sazonal das velocidades do vento no Brasil é influenciada por mecanismos de circulação atmosférica em macro escala. Desta maneira, quando a Zona de Convergência Intertropical está localizada ao sul do Equador durante o verão e outono, os ventos tendem a serem mais lentos e suaves comparados com os períodos de inverno e primavera. Esta explicação pode estar relacionada a uma subestimativa das tendências do componente $u$, conforme apontado em McVicar et al. (2008), geralmente associado ao método de assimilação de dados utilizado nos conjuntos de reanálises ou na parametrização dos modelos aplicados.

Em termos de variabilidade das séries, as séries observadas possuem um desvio padrão médio de $0,64 \mathrm{~m} / \mathrm{s}$, reproduzido em termos médios no ERA5 $(0,70 \mathrm{~m} / \mathrm{s})$ e MERRA-2 $(0,61 \mathrm{~m} / \mathrm{s})$ e subestimado no GLDAS $(0,56 \mathrm{~m} / \mathrm{s})$. Com relação a variabilidade mensal da velocidade média do vento, observa-se que os ERA5 e o GLDAS 2.1 conseguiram representar a variabilidade inerente das séries observadas nas 5 zonas climáticas do país (Fig. 3) e uma tendência de superestimativa das velocidades médias diárias dos produtos de reanálise para todas as zonas climáticas brasileiras, com exceção do ERA5 


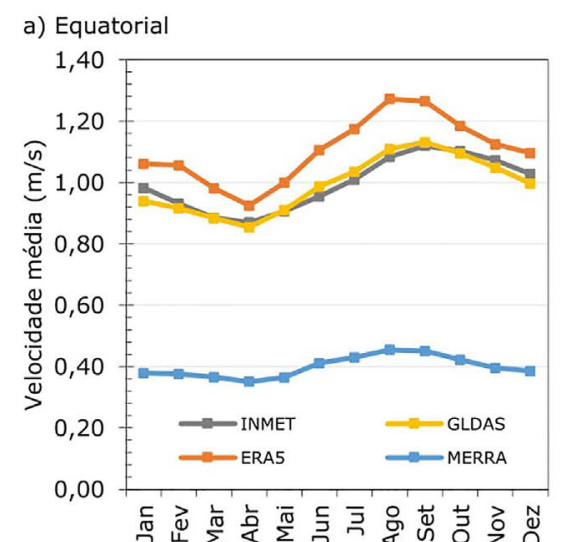

b) Temperado

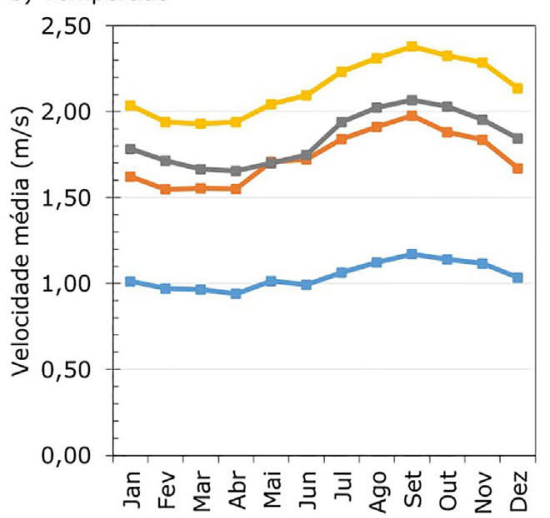

d) Tropical Nordeste Oriental

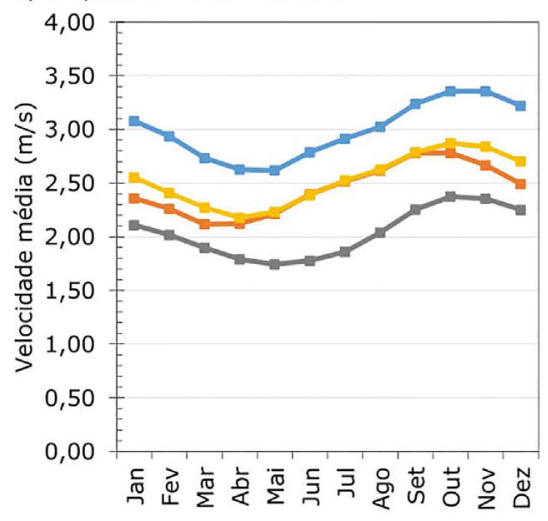

e) Tropical Zona Equtorial

c) Tropical Brasil Central

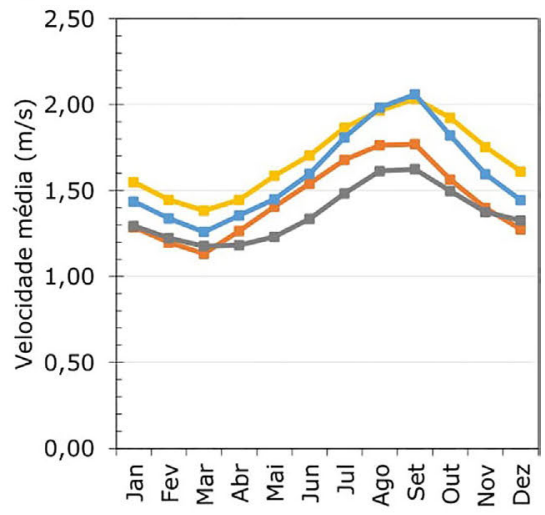

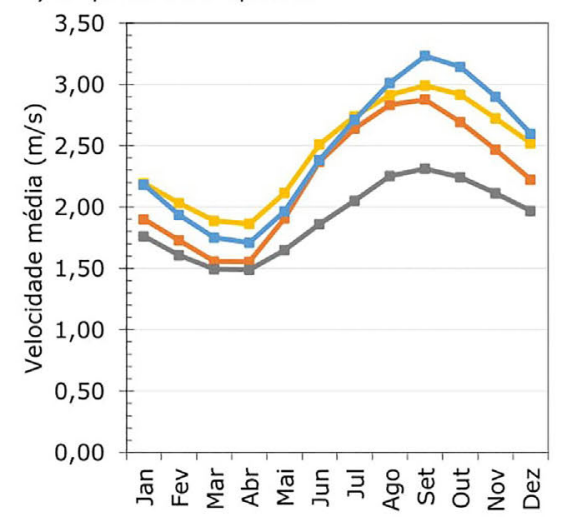

Figura 3 - Velocidade média do vento a $2 \mathrm{~m}(\mathrm{~m} / \mathrm{s})$ observada e dos produtos de reanálises em base mensal.

para a zona Temperado. Os maiores desvios positivos são observados para as estações nas zonas climáticas Tropical Zona Equatorial $(15 \%, 22 \%$ e $23 \%$ para ERA5, GLDAS 2.1 e MERRA-2) e Tropical Nordeste Oriental (16\%, 19\% e $32 \%$ para ERA5, GLDAS 2.1 e MERRA-2). Para as zonas Equatorial e Temperado, o MERRA2 apresenta velocidades médias inferiores $(0,40 \mathrm{~m} / \mathrm{s}$ e $1,05 \mathrm{~m} / \mathrm{s}$, respectivamente) aos dados observados $(1,00 \mathrm{~m} / \mathrm{s}$ e $1,85 \mathrm{~m} / \mathrm{s}$, respectivamente).

Regionalmente, observam-se ligeiras diferenças entre as médias observadas para as séries e os dados das reanálises, sendo que as diferenças mais evidentes são com relação ao MERRA-2 (Fig. 4) em função da limitação da resolução espacial em reproduzir a variabilidade espacial da velocidade de vento média para o período 2000-2018. De maneira geral, os 3 produtos conseguiram reproduzir a heterogeneidade espacial média da velocidade do vento no país em termos gerais, considerando que as menores médias são observadas na zona climática Equatorial (i.e. região amazônica). Observa-se ainda uma tendência geral das maiores velocidades de vento estarem associadas as regiões do Nordeste (i.e. zonas Tropical Nordeste Oriental e na transição entre as zonas Tropical Zona Equatorial e Tropical Brasil Central) e na região sul da zona Temperada (i.e. extremo sul do Rio Grande do Sul).

\subsection{Análise das séries temporais}

A avaliação da aderência das séries de dados das reanálises com os dados observados foi realizada considerando os critérios estatísticos MAE, NRMSE, $r$ e Bias. A Fig. 5 apresenta os resultados dos índices por estação, plotados de acordo com o intervalo de ocorrência e sua frequência. De maneira geral, o ERA5 apresenta uma melhor performance ao representar as séries observadas ao apresentar os melhores ajustes considerando as médias de MAE, NRMSE, $r$ e Bias em comparação ao GLDAS $2.1 \mathrm{e}$ MERRA-2.

Com relação ao MAE, no ERA5 obteve-se uma diferença média absoluta inferior a $0,3 \mathrm{~m} / \mathrm{s}$ entre os valores de velocidade preditos em base diária e observados para 336 estações meteorológicas analisadas (65\% do total de estações), comparativamente com 230 no GLDAS 2.1 (44\%) e 164 no MERRA-2 (30\%). Tal cenário é evidenciado pela distribuição dos valores de $M A E$ na Fig. 5, possuindo uma maior concentração em valores baixos e uma menor amplitude para o ajuste com o ERA5 comparativamente com os outros produtos. A performance do ERA5 na simulação das séries observadas também foi amplamente superior ao GLDAS 2.1 e MERRA-2 considerando o NRMSE $(0,14 \mathrm{~m} / \mathrm{s}, 0,19 \mathrm{~m} / \mathrm{s}$ e $0,2 \mathrm{~m} / \mathrm{s}$, respectivamente, em média). Estas menores diferenças no NRMSE para o ERA5 tornam- 


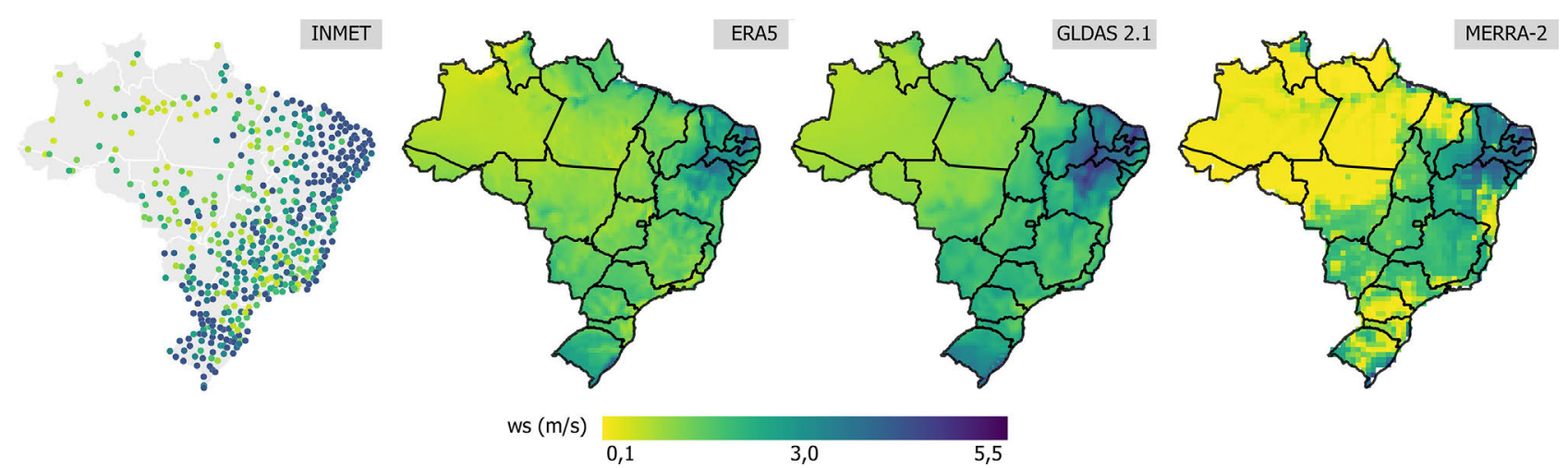

Figura 4 - Velocidade média do vento a $2 \mathrm{~m}(\mathrm{~m} / \mathrm{s})$ para o período de 2000-2018.
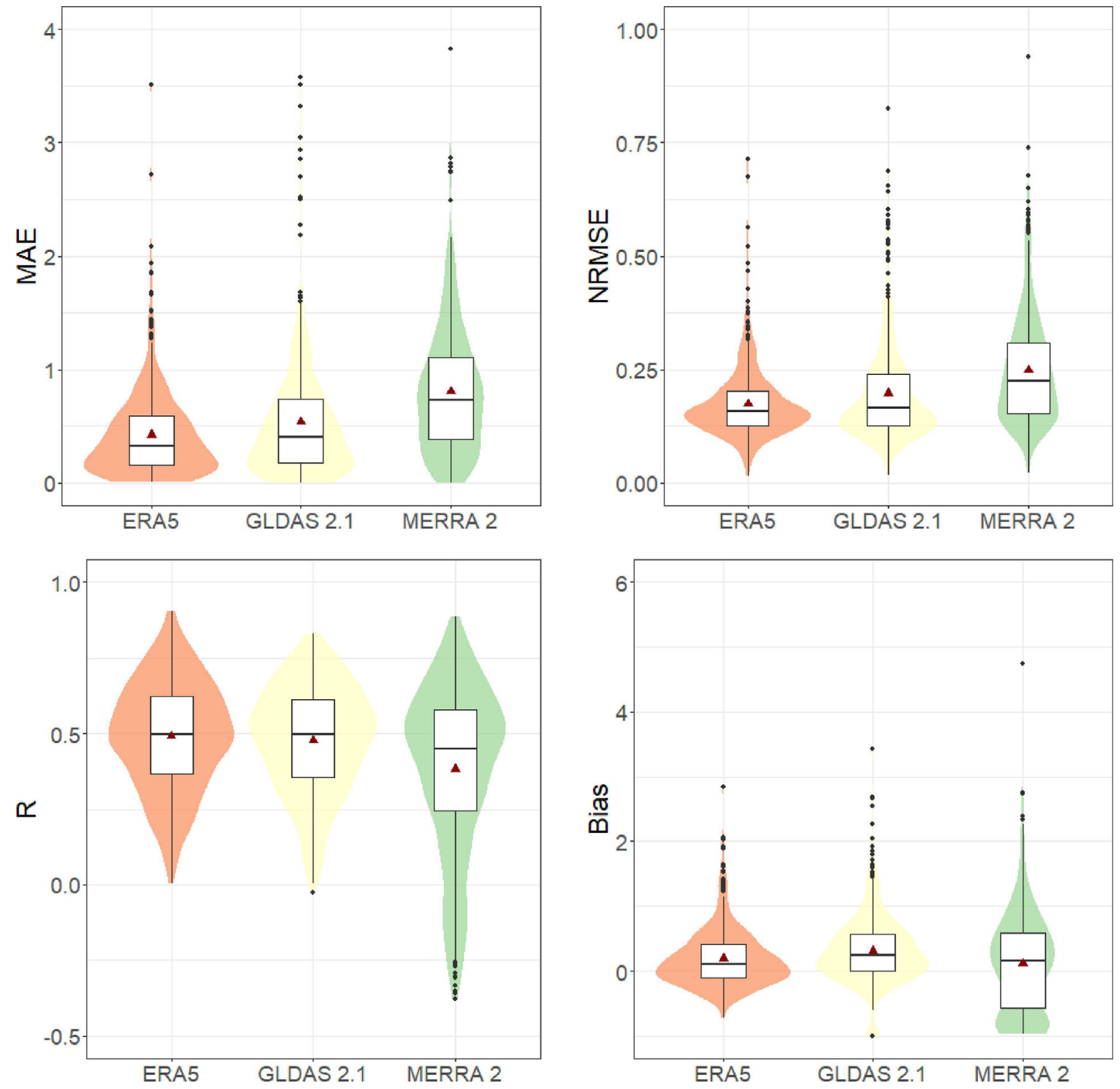

Figura 5 - Violin-plot dos índices MAE, NRMSE, r e Bias, utilizados para avaliação entre os dados observados e dos produtos de reanálises. 
se relevantes considerando que maiores desvios são indesejáveis com relação aos dados observados, uma vez que o valor do NRMSE aumenta proporcionalmente à magnitude da variância da distribuição de frequência dos erros.
Como observado na Fig. 6, os maiores valores de NRMSE para o GLDAS 2.1 e o MERRA-2 foram obtidos para as estações meteorológicas nas zonas Tropical Zona Equatorial e Tropical Nordeste Oriental. Evidencia-se,
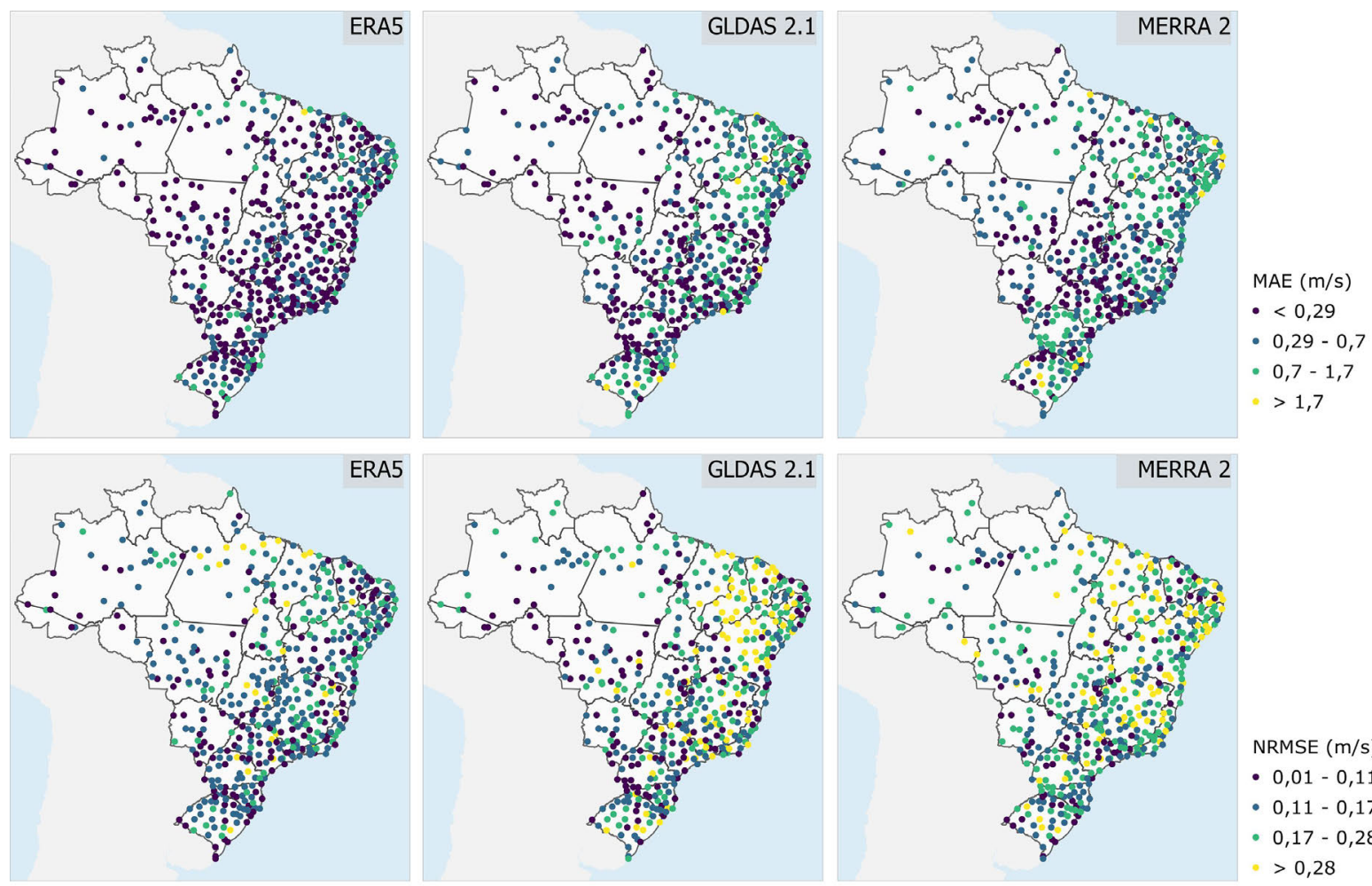

NRMSE $(\mathrm{m} / \mathrm{s})$

- $0,01-0,11$

- $0,11-0,17$

- $0,17-0,28$
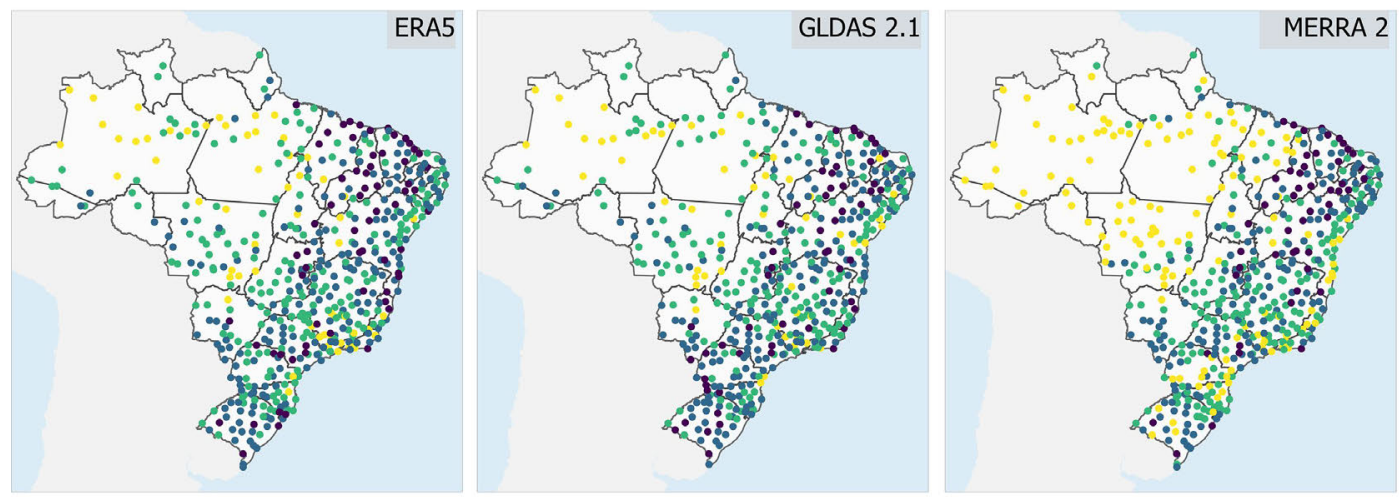

$>0,28$
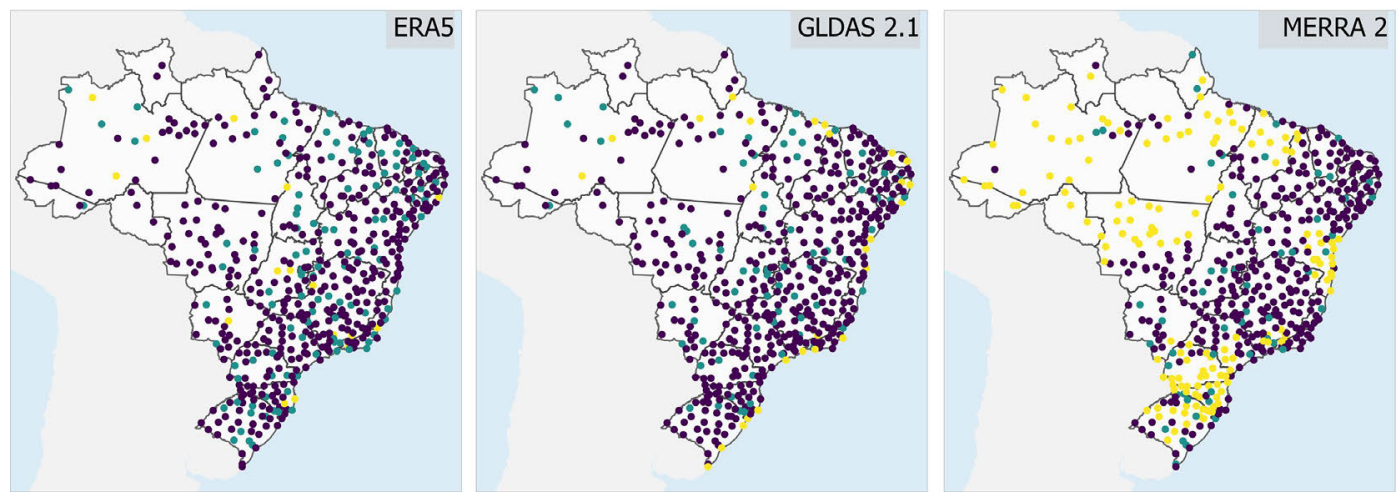

$r(-)$

$>0,25$

- $0,25-0,5$

- $0,5-0,7$

- $>0,7$

Figura 6 - Performance dos produtos de reanálise por estação meteorológica de acordo com os índices utilizados para avaliação. 
portanto, uma limitação dos produtos na representação de regiões com maiores tendência de escoamento atmosférico em velocidades elevadas. Conforme classificação dos regimes de vento do Brasil (Amarante et al., 2001), estas regiões (i.e. litorâneas Nordeste/Norte e Nordeste/Sudeste e zona de elevações Nordeste-Sudeste) são controlados primariamente por brisas terrestres e marinhas e alísio de leste no litoral Norte-Nordeste (Zona Litorânea), com um incremento nas velocidades médias na porção central da região Nordeste. Mais ao Sul, esta região ainda sofre um bloqueio atmosférico de escoamento leste-nordeste, criando condições propícias para a aceleração do ar. Já as áreas de elevações também apresentam velocidades médias altas dentro do contexto brasileiro, com uma ênfase em condições locais de relevo que controlam o escoamento atmosférico.

Considerando a correlação obtida entre as séries observadas e das reanálises, ERA5 e GLDAS 2.1 performaram de maneira ligeiramente similar e superior ao MERRA-2, apresentando 258 estações com $r>0,5$ e 65 com $r>0,7$ e 249 estações com $r>0,5$ e $57 \operatorname{com} r>0,7$, respectivamente. A correlação obtida entre as séries observadas e as reanálises possui uma clara regionalização. Ao observar a distribuição espacial dos ajustes, nenhum dos produtos performou razoavelmente na representação das séries na região Amazônica. Esta região apresenta gradientes fracos de pressão e um elevado atrito superficial causado pela rugosidade da vegetação densa (Amarante et al., 2001) resultando nas menores velocidades de vento média sazonalmente e anualmente para o país (Gilliland e Keim, 2017) nas estações localizadas na zona Equatorial ( $w s<1 \mathrm{~m} / \mathrm{s}$ para os dados observados).

Com relação ao viés, indicado pelo Bias, 466 estações possuíram desvios positivos no ERA5, sendo que 113 apresentam desvios absolutos inferiores a $25 \%$. 54 estações apresentaram desvios negativos com relação aos dados do ERA5. O GLDAS 2.1 apresentou um desvio positivo médio para 477 estações sendo que 69 apresentam desvios absolutos inferiores a $25 \%$. O MERRA-2 apresentou uma distribuição com tendência bimodal do viés, conforme identificado na Fig. 5, onde 352 estações apresentaram desvios positivos e 169 estações com desvios negativos, sendo que 53 estações apresentam desvios absolutos inferiores a $25 \%$. Além disso, não se observa um efeito regional nas tendências de superestimativa ou subestimativa das reanálises.

A interpretação dos parâmetros da distribuição de Weibull $(k$ e $c)$ permite indicar a dispersão dos valores com relação à média (i.e. forma do histograma) e o comportamento geral da distribuição de frequência das séries em termos de escala de dados (i.e. diferença entre valores mínimos e máximos de velocidade de vento). Desta maneira, espera-se que um ajuste ideal entre os parâmetros obtidos das séries observadas e simuladas pela reanálise estejam próximas da linha 1:1 (Fig. 7). Para as estações analisadas, os melhores ajustes entre os parâmetros $k \mathrm{e} c \mathrm{e}$ os desvio padrão das séries foram obtidos para o ERA5. Em linhas gerais, as séries simuladas pelas reanálises possuem uma tendência maior de ajuste em termos da escala da distribuição e uma correlação fraca em termos de forma. Ademais, os desvios observados entre os parâmetros obtidos das séries (i.e. distribuição de frequência) podem estar relacionados a diferenças entre as condições de topografia local, especialmente para regiões de relevo complexo, e sua modelagem nos produtos de reanálise devido a sua resolução espacial (Kaiser-Weiss e Kaspar, 2015) ou ainda as próprias condições microclimáticas de entorno (Gillilad e Keim, 2017).

De maneira geral, estes resultados obtidos na comparação das reanálises com as estações meteorológicas no Brasil vão ao encontro de outros estudos que relatam uma performance limitada de diversos conjuntos de reanálises em reproduzir as tendências das velocidades de vento superficiais com base em dados na China (Zhang et al., 2019; Yu et al., 2019), EUA (Rose e Apt, 2016), Holanda (Smits et al., 2005), Portugal (Carvalho et al., 2014) e Austrália (McVicar et al., 2008).

Destaca-se ainda que a assimilação dos dados observados nos conjuntos de reanálises pode aumentar a sua performance em reproduzir os dados de vento em escala local / regional (Yu et al., 2019), conforme identificado em Kaiser-Weiss e Kaspar (2015) para estações na Alemanha. Além disso, a proposição de fatores de correção com base em dados observados ou métodos empíricos para melhora da performance das reanálises ou das simulações derivadas a partir de suas variáveis (i.e. Staffell e Pfenninger, 2016; Mao e Wang, 2017; Paredes et al., 2018; Rose e Apt, 2019; Ricard e Anctil; 2019) podem representar um importante avanço para a diminuição de incertezas quanto ao uso de dados de velocidade de vento oriundos das reanálises.

\section{Conclusões}

Este estudo apresentou uma avaliação dos dados de velocidade de vento superficial $(2 \mathrm{~m})$ de reanálises climáticas considerando resolução temporal diária e espacial inferior a $1^{\circ}$ (i.e. ERA5, GLDAS 2.1 e MERRA-2) para o Brasil. Um aspecto chave dos dados de reanálises é a sua plena disponibilidade em termos de cobertura temporal e espacial, de modo que sua avaliação com dados observados é fundamental para a identificação de incertezas no seu uso em estudos que compreendam simulações agrícolas, climatológicas e hidrológicas sobre o território brasileiro.

Quanto aos indicadores de performance avaliados, o ERA5 apresentou um MAE $<0,3 \mathrm{~m} / \mathrm{s}$ para $65 \%$ do total das estações, superior ao observado na análise do GLDAS 2.1 e MERRA-2. O ERA5 e GLDAS 2.1 performaram de maneira ligeiramente similar, e superior ao MERRA-2, em 

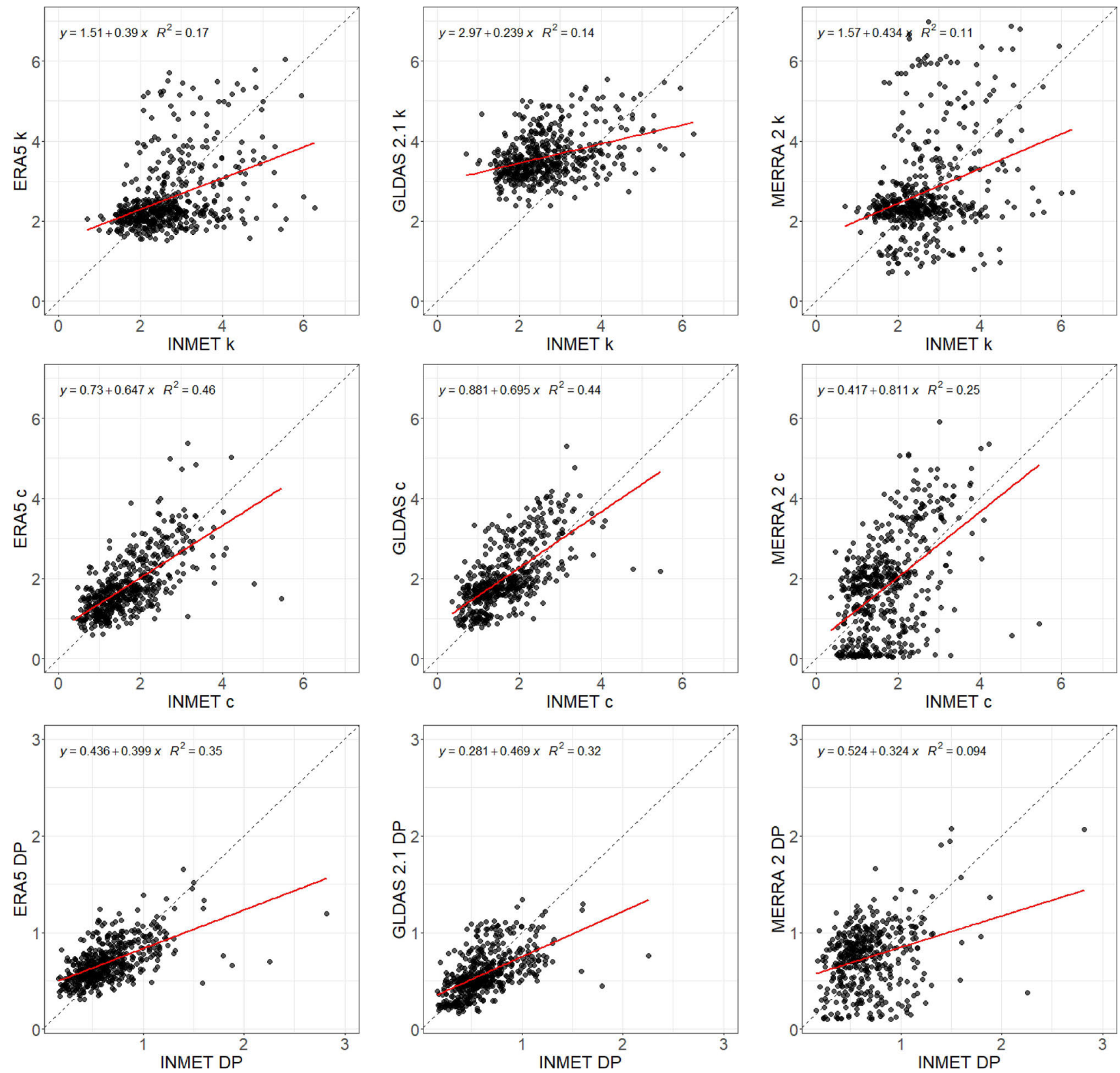

Figura 7 - Parâmetros $k$ e $c$ da função de distribuição de probabilidade do tipo Weibull e desvio padrão para as séries observadas e os produtos de reanálises ERA5, GLDAS 2.1 e MERRA-2.

termos do ajuste de correlação obtida entre as séries observadas e as reanálises, apresentando cerca de $50 \%$ das estações com $r>0,5$ e $10 \%$ com $r>0,7$. O ajuste obtido pelo ERA5 na simulação das séries observadas também foi amplamente superior ao GLDAS 2.1 e MERRA-2 considerando o NRMSE $(0,14 \mathrm{~m} / \mathrm{s}, 0,19 \mathrm{~m} / \mathrm{s}$ e $0,2 \mathrm{~m} / \mathrm{s}$, respectivamente). A análise do viés das séries também indicou que o ERA5 obteve o melhor ajuste aos dados observados, apesar de apresentar um desvio positivo em $90 \%$ das estações avaliadas. Considerando ainda a análise dos parâmetros da distribuição de Weibull, os melhores ajustes em termos de forma e escala das séries, assim como o desvio padrão, também foram obtidos para os dados do ERA5.

Considerando os índices aplicados para a avaliação dos dados de vento dos 3 conjuntos de reanálises e as diferenças obtidas com as séries observadas de 521 estações meteorológicas, os dados do ERA5 apresentaram uma maior acurácia para as 5 zonas climáticas do país em termos de tendências e sazonalidades para o período 20002018. Entretanto, foi identificada uma superestimativa das velocidades médias diárias do ERA5, sobretudo nas zonas climáticas Tropical Zona Equatorial e Tropical Nordeste Oriental. Por fim, destaca-se que os índices obtidos na 
análise indicam que os produtos de reanálise climática avaliados possuem uma tendência de superestimar os valores médios de velocidade de vento superficial para o Brasil, apresentando incertezas e limitações quanto ao seu uso em análises e simulações diversas.

\section{Agradecimentos}

Os autores agradecem ao Projeto "ANA/UFPR - Pesquisa e Inovação em Hidrologia Aplicada", financiado pela Agência Nacional de Águas e Saneamento Básico, pelas bolsas de estudos recebidas.

\section{Referências}

ALLEN, R.G.; PEREIRA, L.S.; RAES, D.; SMITH, M. Crop Evapotranspiration - Guidelines for Computing Crop Water Requirements . FAO Irrigation and Drainage Paper 56. FAO, Rome, 1998.

AMARANTE, O.A.C.; BROWER, M.; ZACK, J.; SÁ, A.L. Atlas do Potencial Eólico Brasileiro, 2001.

AMINI, M.A.; TORKAN, G.; ESLAMIAN, S.; ZAREIAN, M.J.; ADAMOWSKI, J.F. Analysis of deterministic and geostatistical interpolation techniques for mapping meteorological variables at large watershed scales. Acta Geophysica, v. 67, n. 1, p. 191-203, 2019.

ANGELIS-DIMAKIS, A.; BIBERACHER, M.; DOMINGUEZ, J.; FIORESE, G.; GADOCHA, S.; GNANSOUNOU, E., GUARISO, G.; KARTALIDIS, A.; PANICHELLI, I.P.; ROBBA, M. Methods and tools to evaluate the availability of renewable energy sources. Renewable and Sustainable Energy Reviews, v. 15, n. 2, p. 1182-1200, 2011.

BEAUDOING, H.; RODELL, M. GLDAS Noah Land Surface Model L4 3 Hourly 0.25 x 0.25 Degree V2.1. Goddard Earth Sciences Data and Information Services Center (GES DISC), Greenbelt, Maryland, USA, 2020.

BENGTSSON, L.; HAGEMANN, S.; HODGES, K.I. Can climate trends be calculated from reanalysis data? Journal of Geophysical Research: Atmospheres, v. 109, p. 1-8, 2004.

CANNON, D.; BRAYSHAW, D.; METHVEN, J.; COKER, P.; LENAGHAN, D. Using reanalysis data to quantify extreme wind-power generation statistics: A 33 year case study in Great Britain. Renewable Energy, v. 75, p. 767-778, 2015.

CARTA, J.A.; RAMIREZ, P.; VELAZQUEZ, S. A review of wind speed probability distributions used in wind energy analysis case studies in the Canary Islands. Renewable and Sustainable Energy Reviews; v. 13, n. 5, p. 933-55, 2009.

CARVALHO, D.; ROCHA, A.; GÓMEZ-GESTEIRA, M.; SANTOS, C.S. WRF wind simulation and wind energy production estimates forced by different reanalysis: Comparison with observed data for Portugal. Applied Energy, v. 117, p. 116-126, 2014.

CHEN, J.; BRISSETTE, F.P.; CHEN, H. Using reanalysis-driven regional climate model outputs for hydrology modelling. Hydrological Processes, v. 32, n. 19, p. 3019-3031, 2018.

COPERNICUS CLIMATE CHANGE SERVICE (C3S). ERA5: Fifth Generation of ECMWF Atmospheric Reanalyses of the Global Climate. Copernicus Climate Change Ser- vice Climate Data Store (CDS), acesso 20/08/2020, Disponível em https://cds.climate.copernicus.eu/cdsapp\#!/ home, 2017.

DEE, D.; FASULLO, J.; SHEA, D.; WALSH, J.; NATIONAL CENTER FOR ATMOSPHERIC RESEARCH STAFF (eds.). The Climate Data Guide: Atmospheric Reanalysis: Overview \& Comparison Tables, acesso 12/12/ 2016. Disponível em https://climatedataguide.ucar.edu/cli mate-data/atmospheric-reanalysis-overview-comparisontables.

DELHASSE, A.; KITTEL, C.; AMORY, C.; HOFER, S.; AS, D.V.S.; FAUSTO, R.; FETTWEIS, X. Brief communication: Evaluation of the near-surface climate in ERA5 over the Greenland Ice Sheet. The Cryosphere, v. 14, n. 3, p. 957-965, 2020.

FANG, X.; CHEN, Z.; GUO, X.; ZHU, S.; LIU, T.; LI, C.; HE, B. Impacts and uncertainties of climate/ $\mathrm{CO} 2$ change on net primary productivity in Xinjiang, China (2000-2014): A modelling approach. Ecological Modelling, v. 408, p. 110, 2019.

GELARO, R.; MCCARTY, W.; SUÁREZ, M.J.; TODLING, R.; MOLOD, A.; TAKACS, L.; WARGAN, K. The ModernERA retrospective analysis for research and applications, version 2 (MERRA-2). Journal of Climate, v. 30, p. 14, p. 5419-5454, 2017.

GILLILAND, J.M.; KEIM, B.D. Surface wind speed: Trend and climatology of Brazil from 1980-2014. International Journal of Climatology, v. 38, n. 2, p. 1060-1073, 2018.

GORELICK, N.; HANCHER, M.; DIXON, M.; ILYUSHCHENKO, S.; THAU, D.; MOORE, R. Google Earth Engine: Planetary-scale geospatial analysis for everyone. Remote Sensing of Environment, v. 202, p. 18-27, 2017.

HERSBACH, H.; BELL, B.; BERRISFORD, P.; HIRAHARA, S.; HORÁNYI, A.; MUÑOZ-SABATER, J.; SIMMONS, A. The ERA5 global reanalysis. Quarterly Journal of the Royal Meteorological Society, v. 146, p. 1999-2049, 2020.

KAISER-WEISS, A.K.; KASPAR, F.; HEENE, V.; BORSCHE, M.; TAN, D.G.H.; POLI, P.; OBREGON, A.; GREGOW, $\mathrm{H}$. Comparison of regional and global reanalysis near-surface winds with station observations over Germany. Advances in Science and Research, v. 12, n. 1, p. 187198, 2015.

LIMBERGER, L.; SILVA, M.E.S. Precipitação observada na Amazônia brasileira: Comparação entre os dados das redes convencionais e dados da reanálise I do NCEP/NCAR, CRU e GPCC. Revista Brasileira de Climatologia, v. 22, p. 20-37, 2018.

LINDSAY, R.; WENSNAHAN, M.; SCHWEIGER, A.; ZHANG, J. Evaluation of seven different atmospheric reanalysis products in the Arctic. Journal of Climate, v. 27, n. 27, p. 2588-2606, 2014.

MAO, Y.; WANG, K. Comparison of evapotranspiration estimates based on the surface water balance, modified Penman-Monteith Model, and reanalysis data sets for Continental China. Journal of Geophysical Research: Atmospheres, v. 122, n. 6, p. 3228-3244, 2017.

MCVICAR, T.R.; VAN NIEL, T.G.; LI, L.T.; RODERICK, M.L.; RAYNER, D.P.; RICCIARDULLI, L.; DONOHUE, R.J. Wind speed climatology and trends for Australia, 1975-2006: Capturing the stilling phenomenon and com- 
parison with near-surface reanalysis output. Geophysical Research Letters, v. 35, n. 20, p. 1-6, 2008.

MICALICHEN, M.L.M.; DIAS, N.L.C. Análise comparativa da velocidade do vento e da temperatura do ar, entre dados gerados por reanálises meteorológicas e dados observacionais na região de Minas Gerais. Ciência e Natura, v. 40, p. 20-25, 2018.

MUKHERJEE, K.; BHATTACHARYA, A.; PIECZONKA, T.; GHOSH, S.; BOLCH, T. Glacier mass budget and climate reanalysis data indicate a climatic shift around 2000 in Lahaul-Spiti, Western Himalaya. Climatic Change, v. 148, n. 1-2, p. 219-233, 2018.

NEWMAN, A.J.; CLARK, M.P.; CRAIG, J.; NIJSSEN, B.; WOOD, A.; GUTMANN, E.; MIZUKAMI, N.; BREKKE, L.; ARNOLD, J.R. Gridded ensemble precipitation and temperature estimates for the contiguous United States. J. Hydrometeorol., v. 16, p. 2481-2500, 2015.

NIMER, E. Um modelo metodológico da classificação de climas. Revista Brasileira de Geografia, n. 4, p. 59-89, 1979.

PAREDES, P.; MARTINS, D.S.; PEREIRA, L.S.; CADIMA, J.; PIRES, C. Accuracy of daily estimation of grass reference evapotranspiration using ERA-Interim reanalysis products with assessment of alternative bias correction schemes. Agricultural Water Management, v. 210, p. 340-353, 2018.

PES, M.P.; PEREIRA, E.B.; MARENGO, J.A.; MARTINS, F.R.; HEINEMANN, D.; SCHMIDT, M. Climate trends on the extreme winds in Brazil. Renewable Energy, v. 109, p. 110-120, 2017.

PRYOR, S.C.; BARTHELMIE, R.J.; YOUNG, D.T.; TAKLE, E.S.; ARRITT, R.W.; FLORY, D., GUTOWSKI, W.J.; NUNES, A.; ROADS, J. Wind speed trends over the contiguous United States. Journal of Geophysical Research: Atmospheres, v. 114, p. 1-18, 2009.

RAMON, J.; LLEDÓ, L.; TORRALBA, V.; SORET, A.; DOBLAS-REYES, F.J. What global reanalysis best represents near-surface winds?. Quarterly Journal of the Royal Meteorological Society, v. 145, n. 724, p. 32363251, 2019.

RICARD, S.; ANCTIL, F. Forcing the Penman-Montheith Formulation with humidity, radiation, and wind speed taken from reanalysis, for hydrologic modeling. Water, v. 11, n. 6, p. 1214, 2019.

ROCHA, P.A.C.; SOUSA, R.C.; ANDRADE, C.F.; SILVA, M.E.V. Comparison of seven numerical methods for determining Weibull parameters for wind energy generation in the Northeast region of Brazil. Applied Energy, v. 89, n. 1, p. 395-400, 2012.

RODELL, M.; HOUSER, P.R.; JAMBOR, U.; GOTTSCHALCK, J.; MITCHELL, K.; MENG, C.; ARSENAULT, K.; COSGROVE, B.; RADAKOVICH, J.; BOSILOVICH, M.; ENTIN, J.K.; WALKER, J.P.; LOHMANN, D.; TOLL, D. The global land data assimilation system. Bull. Amer. Meteor. Soc., v. 85, p. 381-394, 2004.

ROSE, S.; APT, J. Quantifying sources of uncertainty in reanalysis derived wind speed. Renewable Energy, v. 94, p. 157-165, 2016.

SANTOS, A.T.S.; SILVA, C.M. Seasonality, interannual variability, and linear tendency of wind speeds in the Northeast Brazil from 1986 to 2011. The Scientific World Journal, v. 2013, 2013.

SEDGHI, M.; HANNANI, S.K.; BOROUSHAKI, M. Estimation of Weibull parameters for wind energy application in Iran's cities. Wind and Structures, v. 21, n. 2, p. 203-221, 2015.

SMITS, A.; KLEIN TANK, A.M.G.; KÖNNEN, G.P. Trends in storminess over the Netherlands, 1962-2002. International Journal of Climatology, v. 25, p. 1331-1344, 2005.

STAFFELL, I.; PFENNINGER, S. Using bias-corrected reanalysis to simulate current and future wind power output. Energy, v. 114, p. 1224-1239, 2016.

TAREK, M.; BRISSETTE, F.P.; ARSENAULT, R. Evaluation of the ERA5 Reanalysis as a potential reference dataset for hydrological modelling over North America. Hydrology and Earth System Sciences, v. 24, n. 5, p. 2527-2544, 2020.

UNIYAL, B.; DIETRICH, J.; VU, N.Q.; JHA, M.K.; ARUMÍ, J.L. Simulation of regional irrigation requirement with SWAT in different agro-climatic zones driven by observed climate and two reanalysis datasets. Science of the Total Environment, v. 649, p. 846-865, 2019.

YU, J.; ZHOU, T.; JIANG, Z.; ZOU, L. Evaluation of near-surface wind speed changes during 1979 to 2011 over China based on five reanalysis datasets. Atmosphere, v. 10, n. 12, p. 804, 2019.

ZHANG, R.; ZHANG, S.; LUO, J.; HAN, Y.; ZHANG, J. Analysis of near-surface wind speed change in China during 1958-2015. Theoretical and Applied Climatology, v. 137, n. 3-4, p. 2785-2801, 2019.

License information: This is an open-access article distributed under the terms of the Creative Commons Attribution License (type CC-BY), which permits unrestricted use, distribution and reproduction in any medium, provided the original article is properly cited. 